\title{
ON SAKAGUCHI FUNCTIONS
}

\author{
DING-GONG YANG and JIN-LIN LIU
}

Received 20 May 2002

Let $S_{S}(\alpha)(0 \leq \alpha<1 / 2)$ be the class of functions $f(z)=z+\cdots$ which are analytic in the unit disk and satisfy there $\operatorname{Re}\left\{z f^{\prime}(z) /(f(z)-f(-z))\right\}>\alpha$. In the present paper, we find the sharp lower bound on $\operatorname{Re}\{(f(z)-f(-z)) / z\}$ and investigate two subclasses $S_{0}(\alpha)$ and $T_{0}(\alpha)$ of $S_{S}(\alpha)$. We derive sharp distortion inequalities and some properties of the partial sums for functions in the classes $S_{0}(\alpha)$ and $T_{0}(\alpha)$.

2000 Mathematics Subject Classification: 30C45.

1. Introduction. Let $A$ be the class of functions $f(z)=z+\cdots$ which are analytic in the unit disk $E=\{z:|z|<1\}$. We denote by $S$ the subclass of $A$ consisting of functions which are univalent in $E$. A function $f(z) \in A$ is said to be in the class $S_{S}(\alpha)$ if it satisfies

$$
\operatorname{Re}\left\{\frac{z f^{\prime}(z)}{f(z)-f(-z)}\right\}>\alpha \quad(z \in E)
$$

for some $\alpha(0 \leq \alpha<1 / 2)$. Further, a function $f(z) \in A$ is said to be in the class $T_{S}(\alpha)$ if and only if $z f^{\prime}(z) \in S_{s}(\alpha)$ for some $\alpha(0 \leq \alpha<1 / 2)$. We denote $S_{s}(0)=S_{s}$. Sakaguchi [3] introduced the class $S_{s}$ and proved that $S_{s} \subset C(\subset S)$, where $C$ is the class of close-to-convex functions. The class $S_{s}$ has been studied by several authors (e.g., Stankiewicz [4] and Wu [6]).

For the class $S_{S}(\alpha)$, Owa et al. [2] have proved the following theorem.

THEOREM 1.1. If $f(z) \in S_{s}(\alpha)$ with $1 / 4 \leq \alpha<1 / 2$, then

$$
\operatorname{Re}\left\{\frac{f(z)-f(-z)}{z}\right\}>\frac{2}{3-4 \alpha} \quad(z \in E) .
$$

The following two lemmas were shown by Cho et al. in [1].

LEMMA 1.2. If $f(z)=z+\sum_{n=2}^{\infty} a_{n} z^{n} \in A$ satisfies

$$
\sum_{n=2}^{\infty}\left\{2(n-1)\left|a_{2 n-2}\right|+(2 n-1-2 \alpha)\left|a_{2 n-1}\right|\right\} \leq 1-2 \alpha
$$

for some $\alpha(0 \leq \alpha<1 / 2)$, then $f(z) \in S_{s}(\alpha)$. 
LEMMA 1.3. If $f(z)=z+\sum_{n=2}^{\infty} a_{n} z^{n} \in A$ satisfies

$$
\sum_{n=2}^{\infty}\left\{4(n-1)^{2}\left|a_{2 n-2}\right|+(2 n-1)(2 n-1-2 \alpha)\left|a_{2 n-1}\right|\right\} \leq 1-2 \alpha
$$

for some $\alpha(0 \leq \alpha<1 / 2)$, then $f(z) \in T_{S}(\alpha)$.

In view of Lemmas 1.2 and 1.3, Cho et al. [1] introduced the subclass $S_{0}(\alpha)$ of $S_{s}(\alpha)$ consisting of functions which satisfy inequality (1.3) in Lemma 1.2, and the subclass $T_{0}(\alpha)$ of $T_{S}(\alpha)$ consisting of functions which satisfy inequality (1.4) in Lemma 1.3. It is easy to see that $T_{0}(\alpha) \subset S_{0}(\alpha)$ for $0 \leq \alpha<1 / 2$.

The following theorems (Theorems $1.4,1.5$, and 1.6) are the main results of [1].

THEOREM 1.4. If $f(z) \in S_{0}(\alpha)$ with $0 \leq \alpha<1 / 2$, then

$$
\operatorname{Re} \frac{f(z)}{z}>\frac{1}{2(1-\alpha)} \quad(z \in E)
$$

THEOREM 1.5. If $f(z) \in S_{0}(\alpha)$ with $0 \leq \alpha<1 / 2$, then

$$
\begin{aligned}
|z|-\frac{1-2 \alpha}{2}|z|^{2}-\frac{1-2 \alpha}{3-2 \alpha}|z|^{3} & \leq|f(z)| \\
& \leq|z|+\frac{1-2 \alpha}{2}|z|^{2}+\frac{1-2 \alpha}{3-2 \alpha}|z|^{3}, \\
1-(1-2 \alpha)|z|-\frac{3(1-2 \alpha)}{3-2 \alpha}|z|^{2} & \leq\left|f^{\prime}(z)\right| \\
& \leq 1+(1-2 \alpha)|z|+\frac{3(1-2 \alpha)}{3-2 \alpha}|z|^{2},
\end{aligned}
$$

for $z \in E$.

THEOREM 1.6. If $f(z) \in T_{0}(\alpha)$ with $0 \leq \alpha<1 / 2$, then

$$
\begin{aligned}
|z|-\frac{1-2 \alpha}{4}|z|^{2}-\frac{1-2 \alpha}{3(3-2 \alpha)}|z|^{3} & \leq|f(z)| \\
& \leq|z|+\frac{1-2 \alpha}{4}|z|^{2}+\frac{1-2 \alpha}{3(3-2 \alpha)}|z|^{3}, \\
1-\frac{1-2 \alpha}{2}|z|-\frac{1-2 \alpha}{3-2 \alpha}|z|^{2} & \leq\left|f^{\prime}(z)\right| \\
& \leq 1+\frac{1-2 \alpha}{2}|z|+\frac{1-2 \alpha}{3-2 \alpha}|z|^{2}
\end{aligned}
$$

for $z \in E$.

In the present paper, we improve the above results and find the sharp bounds. 


\section{Main results}

THEOREM 2.1. If $f(z) \in S_{s}(\alpha)(0 \leq \alpha<1 / 2)$, then

$$
\operatorname{Re}\left\{\frac{f(z)-f(-z)}{z}\right\}>4^{\alpha} \quad(z \in E) .
$$

The result is sharp.

Proof. For $f(z) \in S_{s}(\alpha)$, it follows from (1.1) that the function

$$
g(z)=\frac{f(z)-f(-z)}{2}
$$

is an odd starlike function of order $2 \alpha \in[0,1)$, that is, $g(-z)=-g(z)$ and

$$
\operatorname{Re} \frac{z g^{\prime}(z)}{g(z)}>2 \alpha \quad(z \in E)
$$

Since every odd function in $S$ is the square-root transform of some function in $S$, there exists an $h(z) \in S$ such that $g(z)=\sqrt{h\left(z^{2}\right)}$. Further, $h(z)$ is also starlike of order $2 \alpha$, or equivalently

$$
\frac{z h^{\prime}(z)}{h(z)}-1 \prec 2(1-2 \alpha) \frac{z}{1-z}
$$

where the symbol $\prec$ stands for subordination.

Applying a result due to Suffridge [5, Theorem 3] to (2.4), we have

$$
\int_{0}^{z}\left(\frac{h^{\prime}(t)}{h(t)}-\frac{1}{t}\right) d t \prec 2(1-2 \alpha) \int_{0}^{z} \frac{d t}{1-t}
$$

which yields that

$$
\frac{h(z)}{z} \prec \frac{1}{(1-z)^{2(1-2 \alpha)}}
$$

Thus, there exists an analytic function $w(z)$ in $E$ such that $|w(z)| \leq|z|$ and

$$
\frac{h(z)}{z}=\left(\frac{1}{1-w(z)}\right)^{2(1-2 \alpha)} \quad(z \in E)
$$

and hence

$$
\frac{g(z)}{z}=\left(\frac{1}{1-w\left(z^{2}\right)}\right)^{1-2 \alpha} \quad(z \in E) .
$$


With the help of the elementary inequality $\operatorname{Re}\left(t^{\beta}\right) \geq(\operatorname{Re} t)^{\beta}(0<\beta \leq 1$ and $\operatorname{Re} t>$ $0)$, it follows from (2.8) that

$$
\begin{aligned}
\operatorname{Re} \frac{g(z)}{z} & \geq\left\{\operatorname{Re}\left(\frac{1}{1-w\left(z^{2}\right)}\right)\right\}^{1-2 \alpha} \geq\left(\frac{1}{1+\left|w\left(z^{2}\right)\right|}\right)^{1-2 \alpha} \\
& \geq\left(\frac{1}{1+|z|^{2}}\right)^{1-2 \alpha}>\frac{1}{2^{1-2 \alpha}} \quad(z \in E)
\end{aligned}
$$

for $0 \leq \alpha<1 / 2$. This proves (2.1).

To establish the sharpness, we consider the function

$$
f_{0}(z)=\frac{z}{\left(1+z^{2}\right)^{1-2 \alpha}}
$$

It is easy to verify that $f_{0}(z) \in S_{s}(\alpha)$ and

$$
\operatorname{Re}\left\{\frac{f_{0}(z)-f_{0}(-z)}{z}\right\}=2 \operatorname{Re}\left\{\frac{1}{\left(1+z^{2}\right)^{1-2 \alpha}}\right\} \rightarrow 4^{\alpha}
$$

as $z=\operatorname{Re} z \rightarrow 1$. The proof of the theorem is now complete.

REMARK 2.2. This theorem improves and extends Theorem 1.1.

As an immediate consequence of Theorem 2.1, we have the following corollary.

COROLLARY 2.3. If $f(z) \in S_{s}(\alpha)$ with $0 \leq \alpha<1 / 2$ and if $f(z)$ is odd, then

$$
\operatorname{Re} \frac{f(z)}{z}>\frac{1}{2^{1-2 \alpha}} \quad(z \in E) .
$$

The result is sharp with the extremal function $f_{0}(z)$ given by (2.10). In particular, if $f(z) \in S_{s}$ and $f(z)$ is odd, then

$$
\operatorname{Re} \frac{f(z)}{z}>\frac{1}{2} \quad(z \in E)
$$

and the result is sharp.

THEOREM 2.4. If $f(z)=z+\sum_{n=2}^{\infty} a_{n} z^{n} \in S_{0}(\alpha)$ with $0 \leq \alpha<1 / 2$, then for $z \in E$,

(a) the following inequalities hold true:

$$
|z|-\frac{1-2 \alpha}{2}|z|^{2} \leq|f(z)| \leq|z|+\frac{1-2 \alpha}{2}|z|^{2} .
$$

Equalities are attained by the function

$$
f(z)=z-\frac{1-2 \alpha}{2} z^{2} \in S_{0}(\alpha)
$$


(b) the following inequalities hold true:

$$
\begin{aligned}
& 1-2\left|a_{2}\right||z|-\frac{3\left(1-2 \alpha-2\left|a_{2}\right|\right)}{3-2 \alpha}|z|^{2} \\
& \quad \leq\left|f^{\prime}(z)\right| \leq 1+2\left|a_{2}\right||z|+\frac{3\left(1-2 \alpha-2\left|a_{2}\right|\right)}{3-2 \alpha}|z|^{2} .
\end{aligned}
$$

Equalities are attained, for example, by

$$
f(z)=z-a z^{2}-\frac{1-2 \alpha-2 a}{3-2 \alpha} z^{3} \in S_{0}(\alpha)
$$

or

$$
f(z)=z+a z^{2}+\frac{1-2 \alpha-2 a}{3-2 \alpha} z^{3} \in S_{0}(\alpha),
$$

where $0 \leq a \leq(1-2 \alpha) / 2$.

Proof. (a) Writing inequality (1.3) in Lemma 1.2 as

$$
\sum_{n=2}^{\infty}\left[n-\left(1+(-1)^{n+1}\right) \alpha\right]\left|a_{n}\right| \leq 1-2 \alpha,
$$

we arrive at $\sum_{n=2}^{\infty}\left|a_{n}\right| \leq(1-2 \alpha) / 2$. Therefore, it follows from $f(z) \in S_{0}(\alpha)$ that for $z \in E$,

$$
\begin{aligned}
& |f(z)| \geq|z|-|z|^{2} \sum_{n=2}^{\infty}\left|a_{n}\right| \geq|z|-\frac{1-2 \alpha}{2}|z|^{2} \geq 0 \\
& |f(z)| \leq|z|+|z|^{2} \sum_{n=2}^{\infty}\left|a_{n}\right| \leq|z|+\frac{1-2 \alpha}{2}|z|^{2} .
\end{aligned}
$$

(b) Since $n-\left(1+(-1)^{n+1}\right) \alpha \geq n(1-2 \alpha / 3)$ for $n \geq 3$, it follows from (2.19) that

$$
2\left|a_{2}\right|+\left(1-\frac{2 \alpha}{3}\right) \sum_{n=3}^{\infty} n\left|a_{n}\right| \leq 1-2 \alpha
$$

for $f(z) \in S_{0}(\alpha)$. Hence, we deduce that for $z \in E$,

$$
\begin{aligned}
\left|f^{\prime}(z)\right| & \geq 1-2\left|a_{2}\right||z|-|z|^{2} \sum_{n=3}^{\infty} n\left|a_{n}\right| \\
& \geq 1-2\left|a_{2}\right||z|-\frac{3\left(1-2 \alpha-2\left|a_{2}\right|\right)}{3-2 \alpha}|z|^{2}>0, \\
\left|f^{\prime}(z)\right| & \leq 1+2\left|a_{2}\right||z|+\frac{3\left(1-2 \alpha-2\left|a_{2}\right|\right)}{3-2 \alpha}|z|^{2} .
\end{aligned}
$$


REMARK 2.5. Note that $\left|a_{2}\right| \leq(1-2 \alpha) / 2$. Theorem 2.4 is an improvement of Theorem 1.5.

THEOREM 2.6. If $f(z) \in T_{0}(\alpha)$ with $0 \leq \alpha<1 / 2$, then for $z \in E$, the following inequalities hold true:

$$
\begin{gathered}
|z|-\frac{1-2 \alpha}{4}|z|^{2} \leq|f(z)| \leq|z|+\frac{1-2 \alpha}{4}|z|^{2}, \\
1-\frac{1-2 \alpha}{2}|z| \leq\left|f^{\prime}(z)\right| \leq 1+\frac{1-2 \alpha}{2}|z| .
\end{gathered}
$$

The results are sharp with the extremal function $f(z)=z-((1-2 \alpha) / 4) z^{2} \in$ $T_{0}(\alpha)$.

Proof. Let $f(z)=z+\sum_{n=2}^{\infty} a_{n} z^{n} \in T_{0}(\alpha)$. Writing inequality (1.4) in Lemma 1.3 as

$$
\sum_{n=2}^{\infty} n\left[n-\left(1+(-1)^{n+1}\right) \alpha\right]\left|a_{n}\right| \leq 1-2 \alpha
$$

we get $\sum_{n=2}^{\infty}\left|a_{n}\right| \leq(1-2 \alpha) / 4$. From this, we easily have (2.23).

Noting that $f(z) \in T_{0}(\alpha)$ if and only if $z f^{\prime}(z) \in S_{0}(\alpha)$, (2.24) follows directly from Theorem 2.4(a).

REMARK 2.7. Theorem 2.6 improves Theorem 1.6.

THEOREM 2.8. Let $f(z)=z+\sum_{n=2}^{\infty} a_{n} z^{n} \in S_{0}(\alpha)$ with $0 \leq \alpha<1 / 2$, and let

$$
s_{1}(z)=z, \quad s_{m}(z)=z+\sum_{n=2}^{\infty} a_{n} z^{n} \quad(m \geq 2) .
$$

Then for $z \in E$, the following inequalities hold true:

$$
\begin{aligned}
& \operatorname{Re} \frac{f(z)}{s_{m}(z)}>\frac{m+\left(1+(-1)^{m+1}\right) \alpha}{m+1-\left(1+(-1)^{m}\right) \alpha}, \\
& \operatorname{Re} \frac{s_{m}(z)}{f(z)}>\frac{m+1-\left(1+(-1)^{m}\right) \alpha}{m+2-\left(3+(-1)^{m}\right) \alpha} .
\end{aligned}
$$

The results are sharp for each $m \geq 1$.

Proof. Let

$$
\lambda_{n}=\frac{n-\left(1+(-1)^{n+1}\right) \alpha}{1-2 \alpha} \quad(n \geq 2) .
$$

Then $\lambda_{n+1}>\lambda_{n}>1(n \geq 2)$ and it follows from (2.19) that

$$
\sum_{n=2}^{m}\left|a_{n}\right|+\lambda_{m+1} \sum_{n=m+1}^{\infty}\left|a_{n}\right| \leq \sum_{n=2}^{\infty} \lambda_{n}\left|a_{n}\right| \leq 1 \quad(m \geq 2) .
$$


If we let

$$
p_{1}(z)=\lambda_{m+1}\left\{\frac{f(z)}{s_{m}(z)}-\left(1-\frac{1}{\lambda_{m+1}}\right)\right\},
$$

then

$$
p_{1}(z)=1+\frac{\lambda_{m+1} \sum_{n=m+1}^{\infty} a_{n} z^{n-1}}{1+\sum_{n=2}^{m} a_{n} z^{n-1}} \quad(m \geq 2)
$$

and from (2.30), we have

$$
\begin{aligned}
\left|\frac{p_{1}(z)-1}{p_{1}(z)+1}\right| & =\left|\frac{\lambda_{m+1} \sum_{n=m+1}^{\infty} a_{n} z^{n-1}}{2+2 \sum_{n=2}^{m} a_{n} z^{n-1}+\lambda_{m+1} \sum_{n=m+1}^{\infty} a_{n} z^{n-1}}\right| \\
& \leq \frac{\lambda_{m+1} \sum_{n=m+1}^{\infty}\left|a_{n}\right|}{2-2 \sum_{n=2}^{m}\left|a_{n}\right|-\lambda_{m+1} \sum_{n=m+1}^{\infty}\left|a_{n}\right|} \leq 1 \quad(z \in E) .
\end{aligned}
$$

Thus, we conclude that $\operatorname{Re} p_{1}(z)>0(z \in E)$, that is,

$$
\operatorname{Re} \frac{f(z)}{s_{m}(z)}>1-\frac{1}{\lambda_{m+1}} \quad(z \in E) .
$$

This proves (2.27) for $m \geq 2$.

If we take

$$
f(z)=z-\frac{1-2 \alpha}{m+1-\left(1+(-1)^{m}\right) \alpha} z^{m+1} \in S_{0}(\alpha),
$$

then $s_{m}(z)=z$ and

$$
\frac{f(z)}{s_{m}(z)} \longrightarrow \frac{m+\left(1+(-1)^{m+1}\right) \alpha}{m+1-\left(1+(-1)^{m}\right) \alpha}
$$

as $z \rightarrow 1$. Hence, the bound in (2.27) is best possible for each $m \geq 2$.

Similarly, if we put

$$
p_{2}(z)=\left(1+\lambda_{m+1}\right)\left(\frac{s_{m}(z)}{f(z)}-\frac{\lambda_{m+1}}{1+\lambda_{m+1}}\right),
$$

then by (2.30), we deduce that

$$
\begin{aligned}
\left|\frac{p_{2}(z)-1}{p_{2}(z)+1}\right| & =\left|\frac{-\left(1+\lambda_{m+1}\right) \sum_{n=m+1}^{\infty} a_{n} z^{n-1}}{2+2 \sum_{n=2}^{m} a_{n} z^{n-1}-\left(\lambda_{m+1}-1\right) \sum_{n=m+1}^{\infty} a_{n} z^{n-1}}\right| \\
& \leq \frac{\left(1+\lambda_{m+1}\right) \sum_{n=m+1}^{\infty}\left|a_{n}\right|}{2-2 \sum_{n=2}^{m}\left|a_{n}\right|-\left(\lambda_{m+1}-1\right) \sum_{n=m+1}^{\infty}\left|a_{n}\right|} \\
& \leq 1
\end{aligned}
$$

for $z \in E$. From this, we easily have (2.28) for $m \geq 2$. Further, the bound in (2.28) is best possible for the function $f(z)$ given by (2.35). 
For $m=1$, replacing (2.30) by

$$
\lambda_{2} \sum_{n=2}^{\infty}\left|a_{n}\right| \leq \sum_{n=2}^{\infty} \lambda_{n}\left|a_{n}\right| \leq 1
$$

and proceeding as the above, the proof of the theorem is completed.

Letting $m=1$ in Theorem 2.8, we have the following corollary.

COROLlary 2.9. Let $f(z) \in S_{0}(\alpha)$ with $0 \leq \alpha<1 / 2$, then for $z \in E$, the following inequalities hold true:

$$
\begin{aligned}
& \operatorname{Re} \frac{f(z)}{z}>\frac{1+2 \alpha}{2} \\
& \operatorname{Re} \frac{z}{f(z)}>\frac{2}{3-2 \alpha} .
\end{aligned}
$$

The results are sharp.

REMARK 2.10. Inequality (2.40) in Corollary 2.9 is an improvement of Theorem 1.4 for $0<\alpha<1 / 2$ and agrees with Theorem 1.4 for $\alpha=0$.

Using inequality (2.25) instead of (2.19), the following theorem can be proved on the lines of the proof of Theorem 2.8. We omitted the details of its proof.

THEOREM 2.11. Let $f(z)=z+\sum_{n=2}^{\infty} a_{n} z^{n} \in T_{0}(\alpha)$ with $0 \leq \alpha<1 / 2$, and let

$$
\begin{aligned}
& s_{1}(z)=z, \quad s_{m}(z)=z+\sum_{n=2}^{\infty} a_{n} z^{n} \quad(m \geq 2), \\
& \mu_{m+1}=\frac{(m+1)\left[m+1-\left(1+(-1)^{m}\right) \alpha\right]}{1-2 \alpha} \quad(m \geq 1) .
\end{aligned}
$$

Then for $z \in E$, the following inequalities hold true:

$$
\begin{aligned}
& \operatorname{Re} \frac{f(z)}{s_{m}(z)}>\frac{\mu_{m+1}-1}{\mu_{m+1}}, \\
& \operatorname{Re} \frac{s_{m}(z)}{f(z)}>\frac{\mu_{m+1}}{1+\mu_{m+1}} .
\end{aligned}
$$

The results are sharp for each $m \geq 1$, with the extremal function

$$
f(z)=z-\frac{z^{m+1}}{\mu_{m+1}} \in T_{0}(\alpha) .
$$

Theorem 2.11, with $m=1$, leads to the following corollary.

COROLlary 2.12. Let $f(z) \in T_{0}(\alpha)$ with $0 \leq \alpha<1 / 2$. Then for $z \in E$,

$$
\operatorname{Re} \frac{f(z)}{z}>\frac{3+2 \alpha}{4}, \quad \operatorname{Re} \frac{z}{f(z)}>\frac{4}{5-2 \alpha} .
$$

The results are sharp. 
ACKNOWLEDGment. This research is partially supported by the National Natural Science Foundation of China Grant 10171087 and Jiangsu Natural Science Foundation Grant 01KJB110009.

\section{REFERENCES}

[1] N. E. Cho, O. S. Kwon, and S. Owa, Certain subclasses of Sakaguchi functions, Southeast Asian Bull. Math. 17 (1993), no. 2, 121-126.

[2] S. Owa, Z. Wu, and F. Y. Ren, A note on certain subclass of Sakaguchi functions, Bull. Soc. Roy. Sci. Liège 57 (1988), no. 3, 143-149.

[3] K. Sakaguchi, On a certain univalent mapping, J. Math. Soc. Japan 11 (1959), $72-$ 75.

[4] J. Stankiewicz, Some remarks on functions starlike with respect to symmetric points, Ann. Univ. Mariae Curie-Skłodowska Sect. A 19 (1965), 53-59.

[5] T. J. Suffridge, Some remarks on convex maps of the unit disk, Duke Math. J. 37 (1970), 775-777.

[6] Z. Wu, On classes of Sakaguchi functions and Hadamard products, Sci. Sinica Ser. A 30 (1987), no. 2, 128-135.

Ding-Gong Yang: Department of Mathematics, Suzhou University, Suzhou 215006, Jiangsu, China

Jin-Lin Liu: Department of Mathematics, Yangzhou University, Yangzhou 225002, Jiangsu, China

E-mail address: j11iucn@yahoo.com.cn 


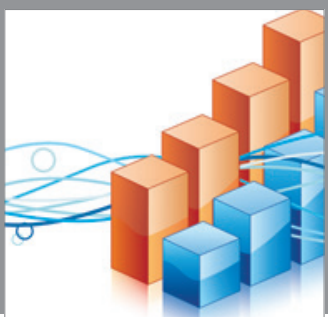

Advances in

Operations Research

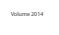

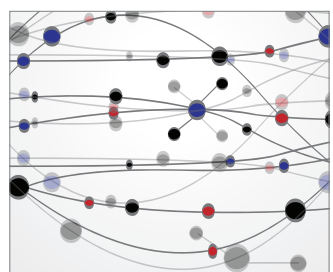

\section{The Scientific} World Journal
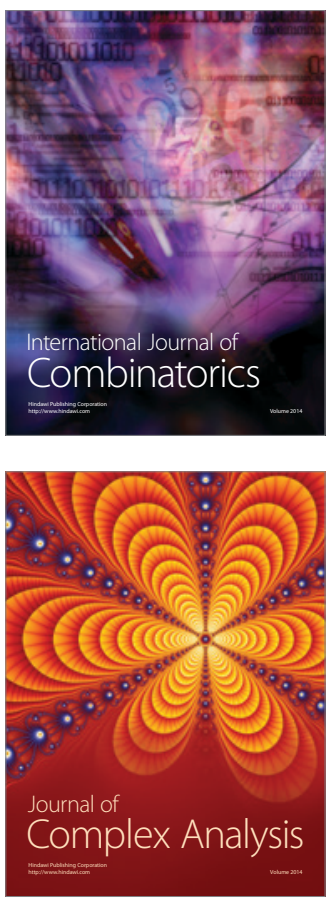

International Journal of

Mathematics and

Mathematical

Sciences
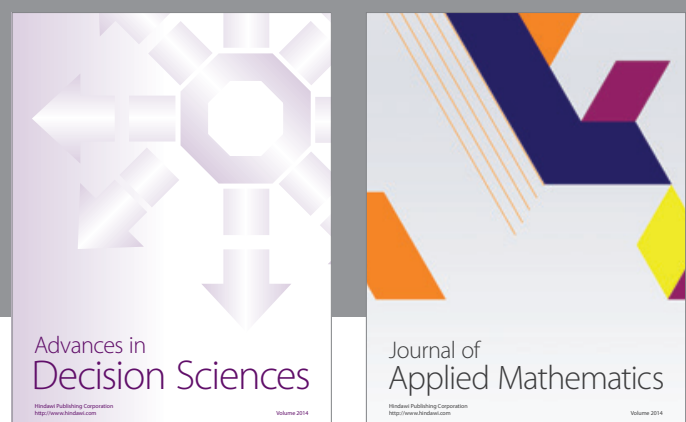

Journal of

Applied Mathematics
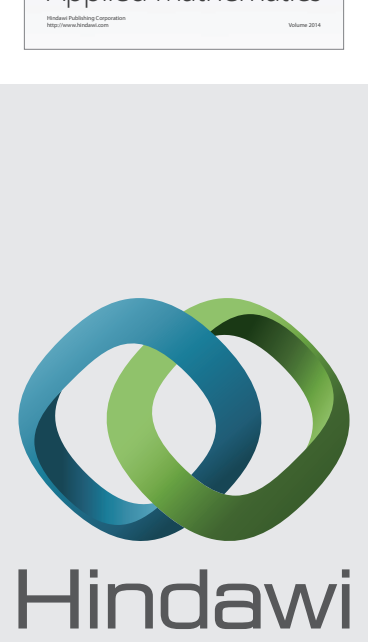

Submit your manuscripts at http://www.hindawi.com
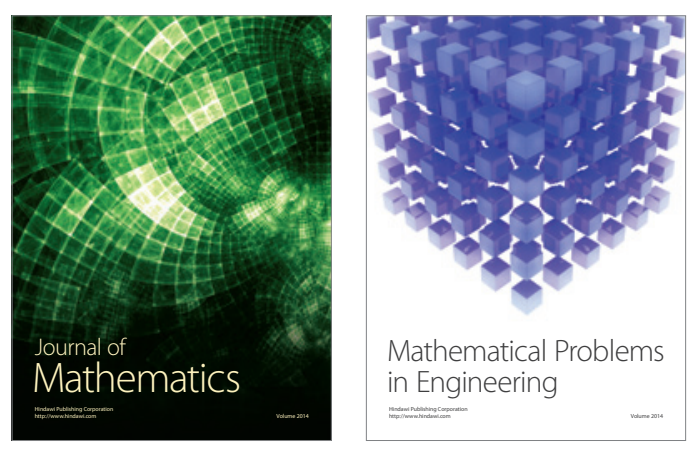

Mathematical Problems in Engineering
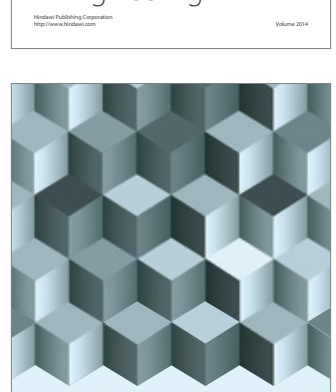

Journal of

Function Spaces
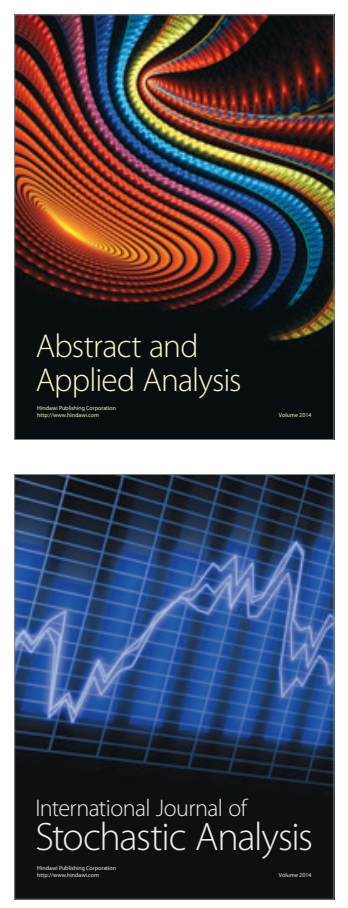

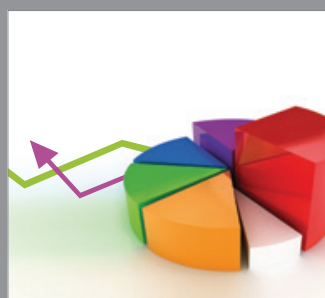

ournal of

Probability and Statistics

Promensencen
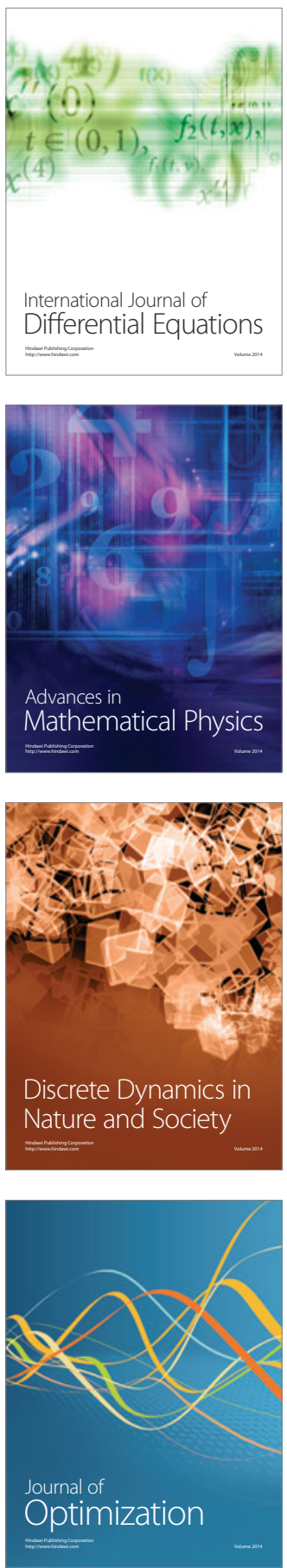